\title{
Bodily map of emotions in Iranian people
}

Running title: [Bodily map of emotions in Iranians]

Molaeinezhad Mitra ${ }^{1,2}$, Scheidt Carl Eduard ${ }^{3}$, Afshar Hamid ${ }^{4}$, Jahanfar Shayesteh ${ }^{2}$ Sohran Fatemeh $^{5}$, Salehi Mina ${ }^{5}$, Goli Farzad ${ }^{6 *}$

1 Mitra Molaeinezhad, PhD, Post doc Researcher in Psychosomatic Medicine, Assistant Professor, Behavioral Sciences Research Center, School of Medicine, Isfahan University of Medical sciences,

2 School of Public health, College of Health Professionals, Central University of Michigan, Mount pleasant, Michigan, USA

Carl Eduard Scheidt, MD, Professor of Psychiatry

${ }^{3}$ Department of Psychosomatic, Albert-Ludwigs-Universitat Freiburg, Freiburg, Germany

Hamid Afshar, MD, Professor of Psychiatry, Fellowship in Psychosomatic Medicine

${ }^{4}$ Psychosomatic Research Center and Departments of Psychiatry, School of Medicine ,Isfahan University of Medical sciences

Shayesteh Jahanfar, PhD, Assistant Professor, Reproductive Health Epidemiologist

2 School of Public Health, College of Health Professionals, Central University of Michigan, Mount pleasant, Michigan, USA

Fatemeh Sohran, Msc in Midwifery Counseling,

${ }^{5}$ Faculty of Midwifery, Nursing and Midwifery school, Islamic Azad University (khorasgan)

Mina Salehi, Msc in Midwifery Counseling,

${ }^{5}$ Faculty of Midwifery, Nursing and Midwifery school, Islamic Azad University (khorasgan)

Farzad Goli ,MD, Fellowship in Psychosomatic Medicine

${ }^{6}$ Head of Danesh-e Tandorosti Institute, Isfahan, Iran and Professor, Faculty Instructor, Energy Medicine University, California, USA

Email: drfgoli@gmail.com

Corresponding addresses: Danesh-e Tandorosti Institute, Isfahan, Iran 


\section{Bodily map of emotions in Iranian people}

\section{Abstract}

Experienced bodily sensation with basic emotions seems to be a universal phenomenon, but cross-cultural differences are expected. A cross-sectional study designed to determine the topographic map of changes across six basic emotions and anxiety in 220 Iranians aged $16-55$ years .The Persian pencil and paper version of the emBODY application was used and validated during the study. The results were analyzed by SPSS software version 20 using generalized linear models (GLM) to determine the relationship between gender and emotion sensation in 5 main body areas and three main facial parts.

The findings supported implicit detection of all basic emotions by participants. Anxiety was the most common reported and anger, fear, sadness, joy, surprise and disgust, respectively were identified basic emotions in both sexes. GLMs were significant ( $P$ $<0.05$ ) for bodily sensation changes in head and neck, upper body, upper and lower limbs, and all three main facial parts. Results also showed a significant gender difference in lower limbs $(\mathrm{P}<0.05)$.

The results suggested the consistency of distinct emotion- triggered bodily sensation maps of Iranians with universal patterns. Implication of the findings for quantification and documentation of the emotion-related similarities and differences of Iranians with the international communities are discussed.

Keywords: Emotion, Cross- Cultural, Culture, Bodily Senses, Gender Difference 


\section{Bodily map of emotions in Iranian people}

\section{Introduction}

There is an accepted notion that the six basic emotions (sadness, anger, surprise, disgust, happiness and fear) have a specific neural basis and are associated with specific body changes in adults (Ekman etal. 1977; Karim S. Kassam , Amanda R. etal.2013). The association between physical changes and various emotions is also reflected in our daily activities; feeling weakness on the knees during fear, or reddening the face with anger, tachycardia with arousal and muscle contraction during anxiety, are examples of personal perception of emotions, which are mostly recognized as universal experiences. Both the classic and modern models of emotional processing believe that subjective experiences of emotions are characterized by emotion-related bodily states that reflects changes in musculoskeletal systems, neuro-endocrine and autonomous nervous systems( Nummenmaa,2014).

The major theories of emotion suggest that a person's subjective feelings of emotion originate from bodily changes perceived by individual (Damasio and Carvalho, 2013; Hietanen, 2016), and the physical perception of emotions has a distinct and wellknown pattern which discussed in various studies (Damasio \& Carvalho, 2013, Philippo, 1997).

While Ekman etal.(1977)believe on universality of the six main emotions, there are few studies which pointed to role of culture in emotions sensation and expression(Matsumoto,1988, 1989, and Dzokoto,2010). Based on this parallel view, emotion is a construct that is not only psychologically and biologically determined, but also influenced by the environment, so cross-cultural differences are expected (Philippo, 1997;Breugelmans 2005). Cultural differences may affect some aspects, including levels of incidence, facial expression, recognition, the nature of experienced emotion (Matsumoto D,1988 , Kitayama S2000a. 2000b)and affect valuation(Tsai JL,2006 ).

Iran is a country made up of heterogeneous populations and different subcultures with common historical and linguistic roots and similarities through Old Persian culture. Iranian nation has some particularities in impressing their experiences as a nation, which distinguished them from the cultures of East Asia and the Arab and Islamic countries of the region (Good, 1977, Malekian, 2013). In general, Iranians are considered socially and interpersonally as sensitive individuals (Good, 1977, Malekian, 2013).

The partial limits of Iranian culture to frankness and openness in expressing expectations, wishes, wills, desires and dissatisfaction as an unwritten rule of respect and politeness, (Novin2009; Dadkhah,2014 ) make individuals to try concealing their 
emotions in some ways or put a partial limit in display rules of emotions in specific situations (Beeman,2001,Malekian, 2013, Pliskin, 1997). The inability of those people to express verbally unpleasant feelings that are not accepted by the community, such as anger and annoyance, (Dadkhah, 2014; Malekian, 2013; Pliskin, 1997) also can contribute to the somatization of emotions in Iranian culture ( Malekian, 2013;Pliskin, 1997).Somatization of symptoms of anxiety and anger such as musculoskeletal pain, heart disease, and digestive problems are frequently found among Iranians (Malekian, 2013;Pliskin,1997;Good, 1977).There is also, an excessive emphasis on grief and sadness in Persian culture and language which is a topic that some linguistic studies have been addressing (Beeman, 2001). Few researchers believe that the Iranian population is more inclined to cry, tears and expressing sadness than other people in the world. They assumed sadness and grief as the most commonly perceived emotion in the Iranians (Beeman, 2001; Surdykowska, 2012, 2014).

One of the problems in recognizing and communicating about emotions in Persian discourse is the use of vague terms related to Persian metaphorical expressions of emotions (Pliskin, 1997, Pirzad Mashak ,2012) and the richness of terminology related to emotions in Persian Language which is impossible to find an equivalent and exact term for those countless expressions (Składankowa , 1993; Surdykowska, 2012). For example, the undifferentiated term called as "Narahati", means "dyphoria". That is used to describe a range of emotions such as depression, illness, anxiety, pain (suffering), disagreement, and discomfort (Pliskin, 1997). As Pliskin (1997), noticed "Iranians express this feeling with non-verbal behaviors such as not speaking and silence and refusing to eat, which is a try to cover or conceal this aspect of their undesirable feeling. On the other hand, this phrase means "annoyance" and anger, and people may not be hiding it“('Pliskin, 1997).

In a constructivist view, the body-part terms used in Persian language are also very ambiguous (Shahshahi, 2008). For example, in Persian, the word "Del" is conceptualized as being either an internal organ and anatomical points of the "heart,"the motor of the body-(Good,1977) and the stomach, and on the other hand, means a container in which emotions, desires, patience, motivation, affection, thoughts and the person's memories (Sharifian,2011;Good, 1977 ; Pirzad Mashak 2012 ). Also, this word is conceptualized as the focus point of personality traits, and mood (Sharifian,2011). Many words used to express emotions come from the combination of the word "Del" with various extensions; e.g. the combination of "delshooreh" and Del Ashoobeh "means anxiety and apprehension, and "delkhooni ", mean sever sorrow and sadness, (Imani, 2018).

To date, limited linguistic studies have been conducted using questionnaires as well as discourse analysis on linguistic concepts and metaphors related to the basic emotions in Iran (Pirzad Mashak, 2012;Beeman, 2001; Riegel‘2017), Which tell us about the inter- 
language and cross -cultural similarities of emotion conceptualization for Iranians with different cultures.

However, no study has been conducted yet, on Iranians' perception of emotion-related bodily changes (ERBCs) or bodily sensation map of emotions (BSMs) using topographic maps and non-verbal questionnaires. The study by Nummenmaa et al.,(2014), using the embody app, is one of the limited studies which eradicates language and semantic barriers in expressing emotions through user-generated bodily sensation maps (BSMs), and helps individuals to create a BSM of how they feel .Hence, make it easier for researchers to detect and classify users emotions based on body maps (García-Magariño,2018).

Moreover, the matter of sex differences in the perception and expression of emotions are also controversial (Pennebaker, 1992, Shahshahani, 2008).It theoretically seems that there are differences between men and women in bodily sensation and understanding relations between bodily sensations and emotional states (Grabauskaite, 2017). The Iranian community places more restrictions on the female body to express emotions, (especially positive emotions i.e. happiness) and gestures in public places, and based on our knowledge, there has not been conducted a study to show the difference between sexes about emotional sensation in Iran. Considering the low threshold of tolerance of Iranian culture for expressing emotions, especially positive emotions such as happiness and more acceptances for expressing feelings such as sadness by women in Iranian culture, differences in physical feelings between sexes can be observed (Shahshahani.2008; Novin,2009, Dadkhah, 2014). But the question that Iranian women and men are different in the perception and bodily sensation of emotions remains unresolved, yet.

Considering the lack of appropriate information about the emotions perceived by the Iranian people and the location of experienced bodily changes associated with basic emotions, further investigations are needed in this regard. In summary, this study has conducted in order to clarify: 1-Whether Iranian samples can differentiate bodily sensation associated with six basic emotions (sadness, anger, disgust, surprise, happiness, and fear) plus anxiety- as a non-basic but prevalent emotion with a wellknown relationship with somatization-? 2- The emotion-specific bodily changes are associated with bodily sensation in which part of the Iranians' body? 3- Which basic emotion is most likely to be perceived with bodily changes among Iranians, 3- Which part of Iranians' bodies reflects more ERBCs? 4- Whether the region that is called as "Del"or heart in Iranian culture reflects the greatest frequency of ERBC? And one of our hypotheses is that the ERBCs are sensitive to sex.

\section{Materials and Methods}


This study is part of a mixed exploratory study with two consecutive qualitative and quantitative phases. The first phase of the study was conducted in a method of discourse and thematic analysis through semi-structured interviews, and the second phase was a quantitative cross-sectional study which has been designed for assessment of ERBCs and BSMs in a sample of Iranian people.

\section{Participants}

The study sample consisted of volunteers selected from a seemingly healthy population; recruited among the visitors to public libraries and parks, students, and referrals to health centers that were referred to periodic examinations and vaccinations for their children. Age of more than 15 years, lack of history of thought disorders, lack of using psychotropic substances, drugs and alcohol and ability to express thoughts and feelings were the inclusion criteria. The number of samples was considered to be at least five cases for each body map points (41 points) according to the rule of thumb (Tabachnik, 1999), and the consecutive sampling was stopped after collecting the minimum number of participants. The research was approved by Isfahan University of medical sciences Ethical Committee (letter no.) and all of the participants gave their informed consents before completion of body map questionnaire.

\section{Data collection tools}

The demographic characteristics of the participants were recorded; e.g. age, occupation, education, history of diseases and drug use, and family history of diseases. All subjects were volunteers agreeing to fill in a Farsi paper -and-pencil version of the emBODY tool designed by the Nummenmaa et al. (2014).

The Farsi emBODY Questionnaire was including two $10 \mathrm{~cm}$ front and back images of the body. During completion of questionnaires, participants were asked to identify and color the bodily regions whose activity they felt to be changed during (Sadness, anger, disgust, happiness, surprise, and fear) plus anxiety. Using colored pencils with distinct colors, the participants had to evaluate intensity of bodily sensation of emotions based on a 5 -point Likert scale (i.e. $1=$ Very mild to $5=$ very severe).

To avoid participants' misperception, along with the consistency between the two genders, the body maps did not reflect any internal organs or forms that represent gender. The reason for not using the emBODY application version was the lack of public access to IOS phones in Iran, besides the need to translate the exact words used for emotions in the app to Persian language. The face and content validity of Persian version was assessed by presenting the preliminary body map questionnaire to ten experts in psychology, psychiatry and linguistic, to assure the correct and valid translation of Persian equivalents were used for each of the emotions. 
Since, concordant BSMs results in the adult population for both techniques shown in previous studies (Nummenmaa et al., 2014; Hietanen, 2016), a memory-based report was used instead of participants' actually inducing emotions.

To simplify the interpretation of the data, we employed a 41 point version of the Michigan Body Map (35 points from the Michigan Pain Map and 6 additional points including the mouth, right and left eyes, forehead, nose and ears, and the left and right jaws replaced with upper and lower Jaw).( Michigan Body map). Then a transparent page was prepared and digitized based on these sections of a standard body template and response sheets screened and compared manually to determine the colored points in each questionnaire. Responses outside the body area were not accounted.

The parts of the BSMs colored by the participants were calculated as the point of perceived emotion $(1=$ yes $)$ and the blank, uncolored points and less than a quarter of the point area accounted as $(\mathrm{No}=0)$. Then the intensity assigned to the perceived emotion at the relevant point in BSMs was manually entered into separate tables related to each emotion. Participants could specify more than one point for each emotion. They could also determine more than one emotion for a specific body point; hence BSMs were screened separately for each emotion.

For better interpretation of the results, the body maps results were divided into 5 general sections i.e. the head and neck, upper trunk, back, upper and lower limbs (Table 1). Furthermore, Using the FAST system (Facial Affect Scoring technique by Ekman group), reported emotion-related facial changes results were divided to three sections (i.e. Facial part $1=$ head and forehead, Facial part $2=$ eyes, and Facial part $3=$ lower part of the face).

\section{Statistical analysis}

Data management and analysis was performed using SPSS version20. Number and frequency used for categorical data and for quantitative data; the mean and standard deviation were used. Independent $\mathrm{T}$ test and Mann Whitney test were used for comparing quantitative data between two groups and General linear Models (GLMs)were used for obtaining sums of squares ,estimated marginal means, testing main effects and producing graphs interactions between gender and perceived emotions (Taylor,2011). P value less than 0.05 was considered as a significant level. The findings about intensity of emotion sensation and profiles of bodily sensation associated with each emotion (e.g., heart beats changes and teeth grinding), which were also completed, will be published elsewhere.

\section{Results}


Response sheets of 220 participants were analyzed (113 women and 107 men). Age ranged from 16 to $55(\mathrm{M}=34.09, \mathrm{SD}=10.87)$.The demographic characteristics of the participants are shown in Table 1.

Table 2 shows the frequency of reported point by point ERBCs associated with each emotion. The first five frequent points where activation was felt for each emotion are listed in the below rows of the table. As we can see in tables 2 and 3, considering the rank order of body sensations for each emotion, comparing point by point hits, all emotions have been associated with changes mostly in the mouth, left chest, forehead, eyes, and hands, respectively. In the 5-part BSM, head and neck, upper trunk, upper and, lower limbs and back were reported as the most common places of emotional perception (tables 3 and4).

The results highlight that the studied population has reported the bodily sensation changes associated with all of the six basic emotions plus anxiety. Figures 1-6 represent the estimated marginal means of ERBCs (total bodily sensation and 5part BSMs) across emotions by sex.

Anxiety was the most reported and among the main six emotions, anger, fear, sadness, happiness, surprise and disgust, respectively were associated with increasing activity in all over the body. We conducted General linear model (GLM) to further investigate the interaction between perceived emotion and gender. As can be seen from the table4,six basic discrete emotion and anxiety significantly predicted mean ratings for total EBRCs among Iranians, $(\mathrm{R} 2=0.93, \mathrm{P}=0.004)$ and variance of the total body sensation was predicted with $92 \%$ accuracy by the emotion perceived. The model for total body sensation also showed the absence of a significant interaction between gender and perceived emotions $(\mathrm{P}>0.05)$ in total ERBCs. When gender was adjusted for emotion perceived, it accounts for only $.043 * 100=4.3 \%$ of the total variance of total bodily perceived emotion so the assumption that regression slopes are similar for both sexes is justified and we accept the results of the original ANCOVA analysis (Table5).

Similarly, the emotion perceived * gender interaction suggested no significant differences between genders in the head \& neck, upper body, upper limbs and facial parts across emotions. Tables 4 displays GLMs and the estimated effect sizes (partial $\eta \mathrm{p}^{2}$ ) of the main effects and interactions for perceived emotions which were significant for ERBCs in head and neck, upper trunk, upper and lower limbs, and all three major facial parts with a cross-gender similarity $(\mathrm{p}<0.05)$. 
Among participants, reported subjective sensation of emotions, anxiety was the most prominent in upper body and sensations were shared across all emotions in this part. Graph 3 shows BSMs is almost identical for both sexes across different emotions in upper trunk (chest and stomach areas). Disgust has been reported in the corresponding parts of the digestive system and mouth.

Anxiety, fear and anger, in the back region are respectively the most perceived emotions reported more by female participants, but the GLM for the interaction between gender and perceived emotions was not significant (Table5). As table 5 shows the GLM for the interaction between perceived emotion and gender failed to significantly explain the variance between bodily sensation in back body part. This finding is difficult to interpret because as graph 4 indicated female participants reported more marginal means in the back area than male participants and it may indicate small sample size and also reflect differences in detection of emotion which have to be accounted for in this interpretation but we did not enter in the analysis because of limitation of our questionnaires and study design.

Anxiety separated early, fear and anger topographies were the most perceived emotions in upper limb (Figure5). In the lower limbs, women perceived anxiety, fear, anger and happiness significantly more than men $(\mathrm{P}<0.05)$ while surprise, disgust and sadness were accordingly the least perceived emotion in the lower limbs. The gender differences in differentiation of body sensations across emotions were found in lower limbs were significant $(\mathrm{p}<0.05)$ and gender accounts about half of the total variance of bodily sensations in lower limbs $(0.551 * 100=5.51 \%)$.

Participants reported anxiety and all six basic emotions in head and neck area, whereas anger (i.e.an approach-oriented emotion) was the most felt-changed emotion in head and neck reflecting in facial areas (Facial parts 1-3 in table 6). The graph in Fig. 7 indicates while men reported relatively more estimated marginal means of ERBCs than women with no significant difference $(\mathrm{p}>0.05)$, anger separated early; happiness, surprise and sad, clustered together followed by disgust separated and anxiety and fear in their own cluster in head and neck for both genders. In the upper part of the face (head, forehead and eyebrows) anger and sadness showed initially the most estimated means, following by happiness, surprise, anxiety, fear. Surprise was separated earlier in facial part2 from other emotions and sadness, anger and happiness clustered then(Fig.8). In facial part 3, participants reported disgust and happiness as the most reported emotions were associated with increasing activity, following by surprise and sadness, anger, which clustered together following by anxiety and fear. There was no significant difference between the two sexes $(\mathrm{P}>0.05)$ (Fig. 9). 


\section{Discussion}

Topographic evaluation of emotionally intensified physical changes can provide a unique tool for emotion studies as well as biomarkers for the detection of emotional disorders (Nummenmaa etal, 2014). Our participants have reported emotional differentiation of body sensations, for all six basic emotions and anxiety and their reported patterns of BSM were consistent with universal patterns(Breugelmans ,2005).This finding is also in agreement with (Nummenmaa etal., 2014) findings which showed that different emotions are associated with topographically distinct and culturally universal bodily sensation.

Contrary to expectations, our data suggests that total EBRCs for all different emotions do not differ between sexes. Graphs in upper trunk was almost the same and emotion perception in back body although showed a greater mean scores for female participants, model failed to reach significance for both sexes. Similarly men reported mean scores in head \& neck and upper limbs which failed to show a significant difference across genders.

Consistent with Nummenmaa etal.'s finding, our results reveal a gender free similarity with universal patterns of BSM. Though in small sample sizes, because of lower statistical power, some of the differences might not been detected, therefore we suggest that issues related to interoception such as emotional awareness- recognition of the connection between body sensations and emotional states and body listening - the tendency to actively listen to the body for insight, which are assumed to be different in both sexes should be further investigated in future studies (Mehling et al. 2012); Grabauskaite , and Griškova-Bulanova,2016) .

Nonetheless, we found differences in the lower limb when comparing males and females in ERBCs especially for fear, anxiety and anger. Bear in mind, the generalizability of "weakening in the knees" as one of the most common bodily sensation with fear, anger (Breugelmans ,2005) and anxiety ,across studies and crosscultural samples, it is not surprising that these emotions reported as the most perceived emotions in lower limb. But interpretation for gender differences is more difficult. These differences can be explained in part by sex differences in noticing and emotional awareness (Mehling et al. (2012);Grabauskaite , and Griškova-Bulanova,2016 )with considering other potential sources of differences such as; cultural meaning of the body sensation (Breugelmans ,2005), gender stereotypes and social desirability of this emotional sensation pattern( Zargar, etal, 2012). Therefore, these factors could be suggested for further investigation in future studies. 
Anxiety and anger were the most reported emotions associated with enhanced sensations all over the body with no significant emotion* gender interaction. The most striking result to emerge from the data is that ,unlike our expectations and many researchers' idea about pervasive presence of sorrow in Persian culture (Składankowa ,1993 ) ,sadness was not the most prominent emotion through user-generated bodily sensation maps. The prior studies were mostly linguistic studies, have attempted to trace manifestation of sadness in different areas of Iranian daily life using semantic methods and emotional terminology (Składankowa, 1993; Pirzad Mashak, 2012; Beeman, 2001 ;Riegel ،2017).Based on our knowledge, this study was the first research conducted to clarify the reported emotion related BSM (for implicit detection of emotions) independence of emotion related words in Iran. So, one can assume that those high-frequency sadness reports have emerged from stereotypical usage of non exact terms connected with sadness for diverse subtle emotional states by Iranians, with a more extended usage in the past (Surdykowska, 2014).

Another explanation is assuming a transition from sadness to anxiety and anger which may have been occurred in recent years in Iranian population. Considering the interrelations between context, emotions and individual and societal actions in conflict situations (Barsman, 2018) and turning now to the historical evidence of socio-political changes in Iran, much of the anger and anxiety may stem from the catastrophic effects of economic instability during recent years due to prolong sanctions, unpredictability of future and challenges of adaptation to all these events. It may contribute to learned helplessness and non-verbal and somatic modes of emotional expression (Malekian, 2012; Hosseini, 2011; Ahmadzadeh, Malekian, \& Maroufi, 2013). Our data must be interpreted with caution though; because the design of study and our questionnaires did not allow us to test whether this association is a culture specific change or no.

Contrary to our expectation" left chest "was not the first in rank order of body sensation across emotions. In Persian literature, there are many words for heart-focused emotions that do not essentially mean more emotional change on the left side of the chest (Good, 1977). In other words, what is called as heart "equal to Del in Farsi" in Persian metaphorical expressions of emotions equals to a large part of the front upper trunk as well as mind and may not necessarily mean the heart (Sharifian,2011). Given that in many cultures there are many words for expression of the emotional state. Many of these terms are just metaphors and stereotypical bodily responses associated with emotions (Nummenmaa etal, 2014) and may not necessarily reflect physiological changes associated with different emotions (e.g. the word butterfly in the stomach (Nummenamaa, 2014, Heelas, 1986).

However, a careful comparison cannot be made until a detailed study is done to clarify what specific bodily sensations are related to the expression of emotions through experiments using emotion induction techniques (Nummenmaa etal. 2014). 
Moreover, the participants' reports of the most emotional perception in the mouth area may reflect the people's verbal culture at this point of the world. Probably reflecting the pattern of emotion suppression in Iranian culture in presence of unwritten rules of politeness which sometimes prohibited Iranians from verbally expression of unpleasant feelings, they report an increased amount of feeling and increased pressure in their mouth area (i.e. Feeling pressure in teethes and teeth grinding during anger- which is not specified in this article). In recent decades, few Iranian researchers have attempted to explore possible causes of intensified range of state-trait anger expression and aggression in Iranian community (Lake and Khal'atbari 2011, Shokouhi 2011, Khodayari fard 2011, Asghari Moghadam 2009, Moheba and Ram 2010, Kazemi, 2017, Shahsavarani and Noohi 2014), However, with a small sample size and in absence of any well-designed epidemiological study about prevalence of anger in Iranians, caution must be applied.

Considering several devastating social and political events (i.e., Iraq-Iran war, natural disasters, and prolong economic and political sanctions against Iran) and in context of vulnerability of Iranians for the somatization of anger and anxiety (Malekian, 2012; Hosseini, 2011), their patterns of anger management and emotion regulation have to be more studied in multidisciplinary researches.

As we know, negative emotions such as anger and fear are integral parts of generation and experience of conflicts (Crawford 2000, 2013, Barsman, 2018). Therefore, although it may be assumed as a very preliminary conclusion based on our findings; we just here want to imply to the conceptual premise for" the long-term role of negative emotions in shaping conflictual interaction and conflict transformation" (Barsman, 2018). We hope that our findings serve as a base for future studies by social psychologists and practitioners in the field to understand more clearly Iranian individuals' emotions and their collective behaviors and to facilitate conflict resolution approaches (Brewer 2010, Brudholm 2008, Chakravarti 2014. Barsman, 2018) intent to re-establishing social cooperation and conducive to build the culture of peace in our population (Barsman , 2018). However, further studies on this topic needs to be undertaken before the association between negative emotions and Iranians' conflictual collective behaviors is more clearly understood.

Sensations of anger in the upper limb as an approach-oriented emotion are in line with universal pattern (Nummenmaa etal 2014, Breugelmans etal, 2005). Anxiety, followed by fear and anger is the most perceived emotion in this area which shows similarity to universal patterns (Breugelmans etal, 2005). 
Concerning Facial patterns, our results were in contrast to the upper face facial expression pattern in Western studies(Jack,2012), which was reported as six "discrete" emotions, though resembles the East Asian emotional expression pattern and shows an overlap for fear, surprise, anger and sadness . However, in our study, anger and sadness were more distinct than other emotions. Although, the studies mentioned were related to emotional expression and not to perceptions of emotions, hence our findings are not exactly comparable to the corresponding studies. But it can be cited as a basis for further studies on comparing emotion expression maps in the Iranian population.

Disgust and happiness were the most common emotions in the lower part of the face (mouth, cheeks and chin) that resemble universal patterns in this respect. Surprisingly, sadness, anger, anxiety, and fear form a cluster that seems to resemble East Asian emotional expression patterns (Jack, 2012). However, the compatibility of these patterns with patterns of emotional expression in the three facial areas should be explored in future studies.

Sensations in the digestive system and mouth region were mainly found in disgust, that consistent with universal sensation patterns (Nummenmaa etal, 2014). This result is also in line with finding of a study by Breugelmans etal, 2005 which showed that "disgust is one of the basic emotions which is appeared to be better differentiated by body sensations across cultures". As graph shows, our participants reported disgust as the least frequent emotion perceived in total bodily sensation map. Considering very little studies conducted about disgust emotion among Iranian individuals, questions raised about "the construct of emotion disgust" among Iranian people(Shams, 2015) and the association between disgust sensitivity and disorders such as obsessive compulsive disorder (Shams, 2015 ) and vaginismus ( Borg,2015). Hence, further experimental investigations are needed.

\section{Study Limitations and Conclusions}

Finally, a number of important limitations need to be considered. First the study sample size was small and was not included different subcultures of Iranian individuals. Second, we could not use the application mode of EmBODY questionnaire hence our results could not exactly compare with EmBODY response profiles. For future studies, therefore we should consider a comparable android or IOS Farsi version of this application permitting from EmBODY manufacturers (Nummenmaa etal, 2014). Third, in absence of a constructivist view (Philippot \& Rime ', 1997 ) and because of the characteristics of questionnaire we used, we did not consider socially constructed components of emotions which people use to express their body sensation ( Ots, 1990,Breugelmans etal, 2005) such as metaphorical and linguistic components of emotions. So, the results of our study may not explicitly reflect the Iranian cultural 
specific variations in body sensation with emotions, which is a subject of further studies.

Although it is a very preliminary study in the field of emotion study but these findings have important implications for developing bodily map of emotions and emotion sensation profile of Iranian people. Overall, the results of this study revealed similarity of Iranians', EBRCs for six basic emotions and anxiety with universal patterns, but considering few differences regarding facial patterns of emotion sensation and mouth as a the top rank point of emotion sensation in our results, further investigation for comprehensive comparing Iranians' emotional sensation patterns with common universal standards are suggested( Breugelmans etal, 2005 , Nummenmaa etal, 2014).

We totally agree with Breugelmans etal, 2005 that "a combination of culture-specific and cross cultural approach" should be applied for exact assessment of association between perceived emotions, embodied feelings and unexplained somatic symptoms.

\section{Conflicts of Interest}

The authors declare that there is no conflict of interest.

This work was supported by the [Isfahan University of Medical sciences] under Grant [number 295256]

\section{References}

Asghari Moghadam, M., Hakimi Rad S, Rezazadeh, H. (2009). Preliminary validation of revised version of State-Trait Anger Expression on student population. Daneshvar-eRaftar, 28, 21-35.[Persian]

Beeman, W. O. (2001). Emotion and sincerity in Persian discourse: accomplishing the representation of inner states. . Int'1. J. Soc. Lang., 148: 31-57.

Bramsen, I.,\& Poder, P. (2018). Emotional Dynamics in Conflict and Conflict Transformation. Berghof Handbook for Conflict Transformation, Online Edition. Berlin: $\quad$ Berghof $\quad$ Foundation. $\quad<\mathrm{http} / /$ www.berghof foundation.org/fleadmin/redaktion/Publications/Handbook/Articles/bramsen_poder_ha ndbook.pdf $>$. First launch 15/02/2018.

Breugelmans, S. M., Poortinga, Y. H., Ambadar, Z., Setiadi, B., Vaca, J. B., Widiyanto P., Philippot, P. (2005).Body Sensations Associated With Emotions in Rara 'muri Indians,Rural Javanese, and Three Student Samples. Emotion, 5( 2), 166-174

Brewer, J. D. (2010). Peace Processes: A Sociological Approach. Oxford: Polity Press. 
Brudholm, T. (2008). Resentment's Virtue -Jean Améry and the Refusal to Forgive. Philadelphia: Temple University Press.

Crawford, N. (2000). The Passion of World Politics - Propositions on Emotion and EmotionalRelationships. International Security, 24(4), 116-156.

Chakravarti S. (2014). Sing the Rage - Listening to Anger after Mass Violence. Chicago: The University of Chicago Press.

Dadkhah, A., Shirinbayan, P. (2014). Emotion Regulation Characteristics Development in Iranian Primary School Pupils. Iranian Rehabilitation Journal, 12(22).

Damasio, A., Carvalho, G.B.(2013).The nature of feelings: evolutionary and neurobiological origins. Nat Rev Neurosci. 2013 Feb;14(2):143-52. doi: 10.1038/nrn3403.

Dzokoto, V . (2010). Different Ways of Feeling: Emotion and Somatic Awareness in Ghanaians and Euroamericans. Journal of Social, Evolutionary, and Cultural Psychology, 4(2), 68-78. Retrieved from www.jsecjournal.com

Ekman, P. (1992). An argument for basic emotions. Cogn Emotion, 6, 169-200.

García-Magariño, I., Chittaro, L., \& Plaza, I. (2018). Bodily sensation maps: exploring a new direction for detecting emotions from user self-reported data. International Journal of Human-Computer Studies, 113, 32-47

Good B., J (1977). The Heart of What's the Matter. The Semantics of Illness in Iran Article in Culture Medicine and Psychiatry, 1(1), 25-58. doi:DOI: 10.1007/BF00114809

Grabauskaite, A., Baranauskas, M., \& Griškova-Bulanova, I. (2017). Interoception and gender: What aspects should we pay attention to? Consciousness and Cognition, 48, 129-137. doi:10.1016/j.concog.2016.11.002

Hietanen, J. K., Glerean, E., Hari, R., \& Nummenmaa, L. (2016). Bodily maps of emotions across child development. Dev Sci, 19(6), 1111-1118. doi:10.1111/desc.12389

Heelas, P. (1986). Emotion talks across cultures. The Social Construction of Emotions, In Harré R(Ed.) (pp. 234-266), Oxford: Blackwell .

Hosseini H., Mokhberi V., Ali Mohammadpour R.,Mehrabianfard M. \& Bali Lashak N. ( (2011). Anger expression and suppression among patients with essential hypertension. International Journal of Psychiatry in Clinical Practice, 15, 214-218. 
Imani A,Rafie A., Amoozadeh M (2018). A socio-constructal analysis of "Del" ( heart) in Persian language in Linguistic literature Lingustic Research Journal, 9(2), 3342. [Persian]

Jack, R. E., Garrod, O. G., Yu, H., Caldara, R., \& Schyns, P. G. (2012). Facial expressions of emotion are not culturally universal. Proc Natl Acad Sci U S A, 109(19), 7241-7244. doi:10.1073/pnas.1200155109

Kassam, K.S., Markey, A.R., Cherkassky, V.L., Loewenstein ,G., Just, M.A. (2013). Identifying Emotions on the Basis of Neural Activation. PLoS ONE 8(6): e66032. https://doi.org/10.1371/journal.pone.0066032

Kitayama, S., \& Markus, H.R. (2000a). The pursuit of happiness and the realization of sympathy: cultural patterns of self, social relations, and well-being. In: Diener E \&Suh EM (Eds.), Culture and subjective well-being. (pp. 113-161). Cambridge, MA: MIT Press

Kitayama, S., Markus, H.R.\& Kurokawa, M. (2000b). Culture, emotion, and wellbeing: good feelings in Japan and the United States. Cogn Emotion, 14, 93-124

Khodayari, fard, M., Lavasani, M.G., Liaghat S. (2011). Standardization of Spielberger's State-Trait Anger Expression Inventory II for Iranian Students.. Tavanbakhshi, 1, 47-56

Lake, M.B. \& Khal'atbari, J. (2011). Effectiveness of anger management program training on control and self-regulation of anger and impulsivity of mothers of male students of primary schools of Rasht.Biquarterly Journal of nursing and midwifery faculties of Guilan province . $63, \quad 61-9 \quad$ [Persian].

Lim, N. (2016) .Cultural differences in emotion: differences in emotional arousal level between the East and the West. Integrative Medicine Research 5, 2, 105-109

Malekian, A., Afshar, H., Ahamadzadeh, Gh. (2014).Cultural Issues in Anxiety Disorders: Some Particularities of the Iranian Culture. Int J Body Mind Culture, 1(1): 54-8.

Matsumoto, D., Kudoh, T., Scherer, K., Wallbott, H. (1988). Antecedents of and reactions to emotions in the United States and Japan. J Cross Cult Psychol, 19, 267286.

Matsumoto, D., \& Ekman, P. (1989). American-Japanese cultural differences in intensity ratings of facial expressions of emotion. Motiv Emot, 13, 143-157. 
Moheba N.,\& Ram U. (2010). Cross-cultural study of stress and anger. Procedia Social and Behavioral Sciences ,5,1765-1769

Mackie, D., Devos, Th., Smith, E.R. (2000). Intergroup Emotions: Explaining Offensive Action Tendencies in an Intergroup Context Journal of Personality and Social Psychology. 79(4):602-16

Mehling, W. E., Price, C., Daubenmier, J. J., Acree, M., Bartmess, E., \& Stewart, A. (2012). The multidimensional assessment of interoceptive awareness (MAIA).PLoS ONE, 7(11), e48230. http://dx.doi.org/10.1371/journal.pone.0048230

Novin, S.,Banerjee, R., Dadkhah, A., \& Rieffe, C. (2009). Self-reported Use of Emotional Display Rules in the Netherlands and Iran: Evidence for Sociocultural Influence. Social Development, 18 (2), 397-411.

Nummenmaa, L., Glerean, E., Hari, R., \& Hietanen, J. K. (2014). Bodily maps of emotions. Proceedings of the National Academy of Sciences, 111(2), 646-651. doi:10.1073/pnas.1321664111

Ots, T. (1990). The angry liver, the anxious heart, and the melancholy spleen. Culture, Medicine, and Psychiatry, 14, 21-58.

Philippot, P., \& Rimé, B. (1997). The perception of bodily sensations during emotion: A cross-cultural perspective. Polish Psychological Bulletin, 28(2), 175-188.

Pliskin, K.L. ( 1992).Dysphoria and somatization in Iranian culture, In Cross-cultural Medicine-A Decade Later [Special Issue]. West J Med ,157,295-300

Pirzad Mashak, Sh. , Pazhakh, A., Hayati ,A. (2012). A Comparative Study on Basic Emotion Conceptual Metaphors in English and Persian Literary Texts.international Education Studies, 5(1), 200-207. doi:10.5539/ies.v5n1p200

Pennebaker, J. W. \& Roberts, T.A. (1992). Toward a His and Hers Theory of Emotion: Gender Differences in Visceral Perception. Journal of Social and Clinical Psychology 11(3), 199-212.

Robinson, M, D. \& Johnson, J,T (1997). Is It Emotion or Is It Stress? Gender Stereotypes and the Perception of Subjective Experience. Sex Roles, 36(3/4), 235-258.

Riegel, M., Moslehi ,A.,Michałowski, J.M., Zurawski, Ł., 'Horvat, M., Wypych, M., Jednoróg, K.\& Marchewka,. A. (2017). Nencki Affective Picture System: CrossCultural Study in Europe and Iran. Front. Psychol. 8:274. doi: 10.3389/fpsyg.2017.00274 
Shahshahani, S. (2008). Body as a means of non-verbal communication in Iran. International Journal of Modern Anthropology/Int. J. Mod. Anthrop. 1 : 1-121 Retrieved from www.ata.org.tn

Shahsavarani A.M. \& Noohi S. (2014).Explaining the Bases and Fundamentals of Anger: A literature Review. International Journal of Medical Reviews 1(4):143-149

Shams, G., Janani, L., Milosevic, I., \& Foroughi, E. (2015). Disgust Emotion and Obsessive-Compulsive

Symptoms in an Iranian Clinical Sample. Psychology, 6( 1), 721-1731. doi:10.4236/psych.2015.613168

Sharifian F. (2011). Cultural Conceptualisations of Del 'Heart/Stomach' in Persian, . In Sharifian F., (Ed.), Cultural Conceptualisations and Language: Theoretical framework and applications. (pp. 157-177). Amsterdam, Netherlands.: John Benjamins Publishing Company.

Shokouhi Yekta M, Zamani, N, Parand A\& Zardkhaneh, SA.(2011).Effectiveness of Anger management training on expression and inhibition of anger in parents. Developmental Psychology: Iranian Psychologists , 26, 137-46 [Persian].

Składankowa , M. (1993). Iran - paradygmaty wyobraźni (II). Acta Asiatica Varsoviensia, 7, 38-39.

Surdykowska, S. ( 2012). Martyrdom and Ecstasy. Emotion Training in Iranian Culture (T. Opalińska, Trans.)Cambridge Scholar Publishing, Newcastle,UK: Newcastle upon Tyne

Surdykowska, S. (2014). The Idea of Sadness. The Richness of Persian Experiences and Expressions. R o c z n i k o r i e $\mathrm{n} \mathrm{t}$ a 1 i s t y c z n y, T., LXVII, Z. 2, s. 68-80.

Tabachnik BG., \& Fidell LS(2001). Using multivariate statistics (Vol. 4th ed. ). MA, USA: Needham Heights: Allyn \&acon.

Taylor A., (2011).Using the GLM Procedure in SPSS. Department of Psychology, Macquarie University Retrieved from http://www.psy.mq.edu.au/psystat/documents/GLMSPSS.pdf

Tsai, JL., Knutson. \& Rung, HH. ( 2006). Cultural variation in affect valuation. J Pers Soc Psychol,90, 288-307. 
Zargar, F., Asgharnejad Farid, A. A., Atef-Vahid, M. K., Afshar, H., Maroofi, M., \& Omranifard, V. (2012). Effect of acceptance-based behavior therapy on severity of symptoms, worry and quality of life in women with generalized anxiety disorder. Iranian journal of psychiatry and behavioral sciences, 6(2), 23-32. 